\title{
DIFICULDADES DAS EMPRESAS \\ BRASILEIRAS PARA FINANCIAR \\ SEUS INVESTIMENTOS EM \\ CAPITAL FÍSICO E EM INOVAÇÃO*
}

\author{
Vicente Lima Crisóstomo
}

RESUMO Este trabalho foi realizado com o objetivo de avançar na pesquisa sobre dificuldades de financiamento da empresa brasileira. Pesquisa-se a existência de tais dificuldades para viabilizar os investimentos em capital físico e também investimentos em inovação. As implicações empíricas são caracterizadas por um modelo de investimento dinâmico, proposto por Bond e Meghir (1994), que assume a presença de custos convexos de ajuste de capital e o objetivo de maximização de valor da empresa. Para uma amostra de empresas não financeiras brasileiras no período 1995-2006 os resultados mostram que a empresa brasileira encara dificuldades de financiamento externo para os investimentos das duas naturezas citadas.

Palavras-chave: dificuldades de financiamento; investimento; investimento em capital físico; investimento em inovação

Código JEL: D92, G32

\section{DIFFICULTIES OF THE BRAZILIAN COMPANIES TO FINANCE THEIR CAPITAL EXPENDITURES AND INNOVATION}

ABSTRACT This work has been done with the purpose of advancing in the research about financing difficulties of the Brazilian company. Such difficulties are researched for capital expenditures as well as for financing innovation activities. The empirical implications are characterized by a dynamic investment model, as proposed by Bond and Meghir (1994), that assumes the presence of convex costs of adjustment

\footnotetext{
* Artigo recebido em 10 de outubro de 2008 e aprovado em 8 de maio de 2009.

** Professor, MSc, Departamento de Contabilidade/Universidade Federal do Ceará, e-mail: vlc@ufc.br
} 
and the objective of firm value maximization. For a sample of non financial Brazilian companies in the period 1995-2006, the results show that the Brazilian firm faces difficulties to finance its capital expenditure as well as innovation with external funds.

Key words: financing difficulties; investment; capital expenditures; investment in innovation 


\section{INTRODUÇÃO}

Não há dúvida sobre a importância do investimento empresarial, seja concretizado em capital físico ou em inovação, tanto em nível micro como macroeconômico, para o desenvolvimento de empresas e países, como a literatura tem destacado ao longo do tempo. Um forte reforço empírico para essa relevância se encontra no trabalho de De Long e Summers (1990), que, usando dados das Nações Unidas e do Penn World Table, confirmam essa importância ao encontrar evidência, para uma amostra de 61 países no período 1960-1985, de que o investimento empresarial tem uma forte associação com o crescimento econômico destes. No entanto, para executar projetos que envolvem investimentos em capital físico e em inovação, a empresa necessita de fundos de que nem sempre dispõe e, por isso, tem de recorrer ao mercado de financiamento externo, seja bancário ou de outra natureza. Esse encontro entre empresa demandante de recursos e mercado não parece ser um encontro em um mercado perfeito, como pensado por Modigliani e Miller (1958), no qual as decisões de investimento e de financiamento seriam independentes. A literatura tem demonstrado teórica e empiricamente que existe um conjunto de problemas, frequentemente referidos como imperfeições de mercado, que dificultam essa relação entre empresa e mercado de financiamento. Essas imperfeições têm mostrado que, de fato, o processo de distribuição de recursos no mercado não ocorre de maneira perfeita (Caballero, 1999; Hubbard, 1998; Stein, 2003). Por um lado, empresas têm dificuldade de obter todos os recursos necessários para desenvolver seus projetos, e, por outro, o mercado tem como objetivo maximizar o retorno sobre o capital que investe nas diversas empresas (Stein, 2003).

As imperfeições do mercado podem originar-se de problemas endógenos associados diretamente à empresa e sua relação com o mercado de financiamento (Myers, 2003). Por outro lado, também se tem observado que fatores exógenos, relacionados com o marco legal e institucional, e o entorno macroeconômico onde se encontra a empresa também parecem afetar a relação entre empresa e mercado (La Porta et al., 1997, 1998; Beck et al., 2003). Este segundo conjunto de fatores tem recebido a atenção de trabalhos que pesquisam distintos países simultaneamente. No entanto, o primeiro conjunto de imperfeições tem sido alvo de pesquisas que investigam 
distintos mercados individual ou conjuntamente. Mais frequentemente, os problemas de assimetria informativa entre empresa e mercado de crédito são tidos como a principal imperfeição de mercado que contribui para a dificuldade de financiamento externo, ou restrição financeira. Esse "conflito informativo" ocasiona um aumento no custo do financiamento externo, como previsto por Akerlof (1970), Stiglitz e Weiss (1981) e Greenwald, Stiglitz e Weiss (1984), levando a um racionamento de crédito para as empresas.

Os conflitos de interesses entre propriedade e controle da empresa, trabalhados detalhadamente por Jensen e Meckling (1976) em sua teoria da empresa, se constituem em outra forma de manifestação típica de imperfeições de mercado que interferem negativamente na política de financiamento da empresa. Esse aspecto está diretamente relacionado com a possibilidade de que haja um desvio do objetivo de maximização do valor da empresa, como destacam Jensen e Meckling (1976) e Crotty (1992). Esse desvio pode ser resultante de comportamento oportunista dos gestores por distintas motivações (Stein, 2003), o que é mais frequente em mercados com propriedade dispersa, como também de aspectos relacionados com a estrutura de propriedade, como quando ocorrem conflitos entre acionistas principais e minoritários, o que é mais característico de mercados com alta concentração de propriedade (Shleifer e Vishny, 1997; Becht e Röell, 1999; Cuervo, 2002, 2004; Morck, Wolfenzon e Yeung, 2005). Uma das consequências desses conflitos pode ser a exacerbação dos problemas de comunicação, ou de assimetria informativa, com o mercado de crédito.

A literatura internacional tem apresentado evidência de que, em função das imperfeições de mercado, empresas, em distintos países, costumam sofrer dificuldades de financiamento externo para seus investimentos em capital físico e em inovação (Hubbard, 1998; Hall, 2005). Considerando a presença dessas imperfeições no mercado brasileiro e seus efeitos na política de financiamento, e diante da evidência internacional, questões trabalhadas na seção 2, propõe-se uma pergunta de pesquisa a que este trabalho pretende responder: "A empresa brasileira tem dificuldade para financiar seus investimentos em capital físico e em inovação?"

Os resultados obtidos nos permitem considerar, com certa segurança, que, de fato, a empresa brasileira encara dificuldades para financiar seus 
projetos de investimento com recursos externos, tanto em capital físico como em inovação.

Para atingir seus objetivos, este trabalho está estruturado em cinco seções, incluindo esta introdução. A seção 1 apresenta uma revisão da literatura relativa à pesquisa sobre dificuldades de financiamento de investimento em capital físico e em inovação e propõe hipóteses de pesquisa para a empresa brasileira. Na seção 2 se apresentam os dados utilizados para a pesquisa bem como a metodologia empregada nesta. Os resultados da pesquisa estão relatados na seção 3. Por fim, as conclusões do trabalho e perspectivas de continuidade são apresentadas na seção 4, que encerra o artigo.

\section{REVISÃO DA LITERATURA E HIPÓTESES DE PESQUISA}

A teoria do investimento evoluiu bastante no século passado, tendo buscado a cada momento distintos fatores capazes de explicar a política de investimento empresarial (Chirinko, 1993a). Mais recentemente, cresceu em importância o papel de fatores financeiros nessa linha de pesquisa como possíveis determinantes do investimento. Apesar de encarar alguns questionamentos, o trabalho de Fazzari, Hubbard e Petersen (1988) foi um marco significativo nesse enfoque da pesquisa ao interpretar a correlação positiva encontrada entre o investimento e o fluxo de caixa como evidência de dificuldade de obtenção de financiamento externo. Alguns autores propuseram que essa relação também podia ser fruto de outros fatores, como, por exemplo, sobreinvestimento (overinvestment), ou a aversão ao alto endividamento (Kaplan e Zingales, 1997; Vogt, 1994; Carpenter, 1994; Gugler, Mueller e Yurtoglu, 2004; Degryse e De Jong, 2006). No entanto, mais recentemente, Alti (2003) e Moyen (2004) avaliam essas possibilidades e concluem que essas interpretações alternativas também podem explicar uma parcela dessa sensibilidade, mas não descartam a interpretação proposta por Fazzari, Hubbard e Petersen (1988).

A interpretação de que a relação positiva encontrada entre fundos internos e investimento é um indicativo de dificuldade de financiamento externo é central em vários trabalhos que detectaram essa dificuldade de crédito em diferentes mercados. Nos Estados Unidos, além de Fazzari, Hubbard e Petersen (1988), devem citar-se Whited (1992), Hubbard, Kashyap e Whited 
(1995) e Hennessy, Levy e Whited (2007) como exemplos. Bond e Meghir (1994) e Goergen e Renneboog (2001) detectaram dificuldades de financiamento externo em empresas do Reino Unido. Em outros países, também se verificou essa dificuldade: Chirinko e Schaller (1995) no Canadá, Harhoff (1998) na Alemanha, Galeotti, Schiantarelli e Jaramillo (1994) na Itália, Gérard e Verschueren (2002) na Bélgica, Lízal e Svejnar (2002) na República Tcheca, e, no Brasil, Moreira e Puga (2000) e Terra (2003).

Bond e Van Reenen (2003) e Hall (2005) comentam que, apesar das diferenças características entre o investimento em capital físico e em inovação, como, por exemplo, mais elevados custos de ajuste de capital, mais altos níveis de risco e de incerteza de resultados e mais longos prazos de investimento, os modelos econométricos de investimento utilizados para capital físico têm sido aplicados também para verificar a existência de dificuldades de financiamento externo de investimentos em inovação. A evidência de existência de dificuldade de crédito externo, ou restrição financeira, para financiar a inovação também foi encontrada em distintos mercados. Este é o caso, por exemplo, de Hall (1992) e Himmelberg e Petersen (1994) nos Estados Unidos, Harhoff (1998) na Alemanha, Brown (1997) e Bond, Van Reenen e Harhoff (2003) no Reino Unido, e Bougheas, Görg e Strobl (2003) na Irlanda.

A pesquisa sobre os dois tipos de investimento, como um todo, difere em aspectos de estratégia de pesquisa e características da amostra. No entanto, o resultado no conjunto é que as dificuldades de obtenção de crédito externo estão presentes, principalmente em empresas consideradas como mais propensas a deparar com esse tipo de situação, como é o caso de empresas menores, ou que pagam menos dividendos, ou ainda mais intensivas em inovação, por exemplo, ou ainda avaliadas de acordo com vários aspectos da sua situação de liquidez a cada ano.

O problema de informação assimétrica é inerente à relação entre empresa e mercado externo de financiamento. Em mercados em desenvolvimento, como é o caso do Brasil, esse problema também está presente. Somando-se a essa questão, devem-se mencionar alguns aspectos do entorno do mercado brasileiro que também favorecem a ocorrência de dificuldades de financiamento externo. Dentre estas se têm as altas taxas de juros (Marques e Fochezatto, 2007), a pouca proteção aos credores e acionistas minoritários 
(La Porta, López-de-Silanes, Shleifer e Vishny, 1997, 1998, 2000), bem como um mercado de capitais ainda pouco desenvolvido, apesar dos avanços alcançados a partir das reformas estruturais dos anos 1990 (Studart, 2000).

Os problemas de assimetria informativa e essas características do mercado brasileiro, somados à evidência internacional na direção de existência de restrições financeiras em distintos mercados, permitem considerar pertinente a proposição de que a hipótese de mercado perfeito, ou seja, ausência de dificuldades de financiamento externo, é descartada no mercado brasileiro e, dessa forma, se prevê que imperfeições do mercado de fato afetam a política de investimento e de financiamento da empresa brasileira. Desse modo, duas hipóteses de pesquisa sobre o financiamento dos investimentos da empresa brasileira são propostas.

Hipótese 1: A empresa brasileira encara dificuldades de financiamento externo para financiar seu investimento em capital físico, o que significa que esse investimento é sensível à disponibilidade de recursos gerados internamente.

Hipótese 2: A empresa brasileira encara restrições financeiras para financiar seu investimento em inovação, o que se traduz em uma correlação positiva entre o investimento em inovação e a disponibilidade de fundos internos.

\section{AMOSTRA E METODOLOGIA}

\subsection{Amostra}

Os dados para a pesquisa foram obtidos da base de dados de Economática e se referem às informações financeiras anuais de empresas não financeiras cotadas na Bovespa no período 1995-2006. O conjunto de informações requerido para a estimação dos modelos forçou o descarte de observações anuais que não dispunham de todas as variáveis necessárias, ou seja, aquelas variáveis relacionadas com as políticas de investimento e de financiamento da empresa. O uso da técnica de dados em painel e método generalizado de momentos (GMM) levou também ao descarte de empresas com menos de seis anos consecutivos de observações válidas. Ao finalizar a seleção dos dados, foi confeccionado um painel de dados não balanceado com 2.808 observações anuais de 289 empresas, cada uma delas com um míni- 


\section{Tabela 1: Distribuição da amostra relativa a gastos de capital por setor}

Para cada amostra, investimento em capital físico e em inovação, apresenta-se o número de observações anuais de empresas de cada setor, em número absoluto e relativo.

\begin{tabular}{|c|c|c|c|c|c|c|c|c|}
\hline \multirow[t]{2}{*}{ Setor da economia } & \multicolumn{4}{|c|}{$\begin{array}{c}\text { Painel A - Amostra para } \\
\text { investimento em capital físico }\end{array}$} & \multicolumn{4}{|c|}{$\begin{array}{c}\text { Painel B - Amostra para } \\
\text { investimento em inovação }\end{array}$} \\
\hline & Obsv. & $\%$ & Empr. & $\%$ & Obsv. & $\%$ & Empr. & $\%$ \\
\hline Administração e empreendimentos & 117 & 4,17 & 12 & 4,15 & & & & \\
\hline Agricultura e produtos de caça/pesca & 18 & 0,64 & 2 & 0,69 & & & & \\
\hline Alimentação e tabaco & 191 & 6,8 & 19 & 6,57 & 168 & 8,30 & 17 & 8,25 \\
\hline Comércio & 103 & 3,67 & 11 & 3,81 & & & & \\
\hline Comunicação e telecomunicações & 110 & 3,92 & 14 & 4,84 & 62 & 3,06 & 7 & 3,40 \\
\hline Construção & 101 & 3,6 & 10 & 3,46 & & & & \\
\hline Editoração & 12 & 0,43 & 1 & 0,35 & 12 & 0,59 & 1 & 0,49 \\
\hline Geração e distribuição de energia & 310 & 11,04 & 34 & 11,76 & 295 & 14,58 & 32 & 15,53 \\
\hline Indústria de instrumentos de precisão & 15 & 0,53 & 2 & 0,69 & 15 & 0,74 & 2 & 0,97 \\
\hline Máquinas industriais & 59 & 2,1 & 5 & 1,73 & 52 & 2,57 & 5 & 2,43 \\
\hline Mineração & 33 & 1,18 & 3 & 1,04 & & & & \\
\hline Outras indústrias & 48 & 1,71 & 4 & 1,38 & 54 & 2,67 & 5 & 2,43 \\
\hline Outros serviços & 48 & 1,71 & 6 & 2,08 & & & & \\
\hline Papel e celulose & 77 & 2,74 & 7 & 2,42 & 59 & 2,92 & 6 & 2,91 \\
\hline Petróleo e gás & 94 & 3,35 & 9 & 3,11 & 93 & 4,60 & 9 & 4,37 \\
\hline Produtos de borracha e plástico & 43 & 1,53 & 4 & 1,38 & 31 & 1,53 & 3 & 1,46 \\
\hline Produtos de madeira e similares & 24 & 0,85 & 2 & 0,69 & 24 & 1,19 & 2 & 0,97 \\
\hline Produtos de vidro e pedra & 59 & 2,1 & 7 & 2,42 & 58 & 2,87 & 7 & 3,40 \\
\hline Produtos elétricos e eletrônicos & 89 & 3,17 & 9 & 3,11 & 69 & 3,41 & 7 & 3,40 \\
\hline Químico & 231 & 8,23 & 22 & 7,61 & 205 & 10,13 & 20 & 9,71 \\
\hline Serviços de hotelaria e similares & 29 & 1,03 & 4 & 1,38 & & & & \\
\hline Serviços de transporte & 51 & 1,82 & 7 & 2,42 & & & & \\
\hline Serviços educacionais & 6 & 0,21 & 1 & 0,35 & & & & \\
\hline Siderurgia e metalurgia & 397 & 14,14 & 41 & 14,19 & 356 & 17,60 & 37 & 17,96 \\
\hline $\begin{array}{l}\text { Serviços de fornecimento de } \\
\text { água e saneamento básico }\end{array}$ & 16 & 0,57 & 2 & 0,69 & & & & \\
\hline Têxtil & 293 & 10,43 & 28 & 9,69 & 277 & 13,69 & 26 & 12,62 \\
\hline Veículos e componentes & 234 & 8,33 & 23 & 7,96 & 193 & 9,54 & 20 & 9,71 \\
\hline Total & 2.808 & 100 & 289 & 100 & 2.023 & 100,00 & 206 & 100,00 \\
\hline
\end{tabular}

mo de 6 e um máximo de 12 observações. As empresas da amostra estão distribuídas em 27 setores da economia, de acordo com a classificação da Bovespa (tabela 1, painel A). Para a pesquisa sobre gastos em inovação utilizou-se uma subamostra desta, na qual se mantiveram os setores que desenvolvem atividades de inovação. Essa subamostra (tabela 1, painel B) está composta por um total de 2.023 observações de 206 empresas distribuídas em 17 setores da economia. 


\subsection{Metodologia}

A contrastação empírica das hipóteses de pesquisa é feita através da estimação de um modelo de investimento baseado na equação de Euler proposto por Bond e Meghir (1994) e também já aplicado bem-sucedidamente com algumas variações em outras pesquisas (Bond, Elston, Mairesse e Mulkay, 2003; Goergen e Renneboog, 2001; Harhoff, 1998). Esse modelo explica os investimentos em capital físico e em inovação em uma relação entre taxas de investimento em períodos sucessivos assumindo o objetivo de maximização de valor da empresa na presença de custos de ajuste de capital simétricos e quadráticos, além de prever a possibilidade de preferência por fonte de financiamento, como proposto pela teoria pecking order (Myers e Majluf, 1984). Assim, o modelo considera que a intensidade de investimento da empresa é explicada pelas expectativas de retorno de investimentos futuros descontados e ajustados pelo impacto de mudanças esperadas em preços da produção, pelos custos de ajuste de capital, pela situação de liquidez da empresa e pelo nível de endividamento.

Para a amostra de empresas selecionada estimaram-se quatro modelos de investimento. O modelo básico, proposto por Bond e Meghir (1994), foi aplicado à amostra correspondente à pesquisa sobre gastos de capital, conforme a equação 1, e uma variação dele foi aplicada para os gastos em inovação (equação 2). Uma variação de cada um desses modelos foi também estimada modificando a variável referente ao endividamento ao quadrado por outra considerando uma relação linear com a dívida bancária. Utiliza-se a dívida bancária por ser a dívida associada mais diretamente com as possibilidades e custos de falência.

$$
\left(\frac{I C F}{K}\right)_{i, t}=\beta_{1}\left(\frac{I C F}{K}\right)_{i, t-1}+\beta_{2}\left(\frac{I C F}{K}\right)_{i, t-1}^{2}+\beta_{3}\left(\frac{F C}{K}\right)_{i, t-1}+\beta_{4}\left(\frac{P}{K}\right)_{i, t-1}+\beta_{5}\left(\frac{D}{K}\right)_{i, t-1}^{2}+\alpha_{i}+\delta_{t}+\varepsilon_{i, t}
$$

A variável dependente $(I C F / K)$ é uma aproximação para medir a intensidade de investimento em capital físico da empresa $i$ no período $t$ e se define como o quociente entre o investimento em capital físico do período $t\left(I C F_{t}\right)$ e o estoque de capital físico produtivo da empresa no início dele $\left(K_{t-1}\right)$. $\mathrm{O}$ investimento em capital físico no período $\left(I C F_{t}\right)$ é calculado como a diferença entre o estoque de capital físico atual $\left(K_{t}\right)$, aproximado pelo ativo 
imobilizado, e o estoque no começo do período ajustado pela dotação à depreciação $\left((1-\delta) K_{t-1}\right)$, onde $\delta$ é a taxa de depreciação econômica. O modelo também incorpora os custos de ajuste de capital que são aproximados pela variável referente à intensidade de investimentos do período anterior ao quadrado (Bond e Meghir, 1994; Lucas, 1967).

As demais variáveis são relativizadas pelo estoque de capital do período: $F C_{t}$ é o fluxo de caixa do período, definido como a soma do lucro líquido e as depreciações; $P_{t}$ é o nível de produção (output), aproximado pelo faturamento da empresa; $D_{t}$ é a dívida bancária da empresa; $\alpha_{i}$ é o termo de erro relacionado com efeitos fixos de empresas, ou seja, fatores não observáveis específicos de empresa e constantes ao longo do tempo; $\delta_{t}$ é o termo de erro associado a questões temporais; e $\varepsilon_{i, t}$ se refere a erros aleatórios.

Uma variação desse modelo é estimada para a amostra referente aos gastos em inovação com a argumentação de que a relação investimento-vendas é mais estável ao longo do tempo (Goergen e Renneboog, 2001). A pesquisa sobre investimento em inovação mais frequentemente relativiza as variáveis de investimento e financeiras pelo faturamento, como fazem Audretsch e Weigend (2005); Bond, Van Reenen e Harhoff (2003); Hall, Mairesse, Branstetter e Crepon (1999), Mulkay; Hall e Mairesse (2001); e Tribo, Berrone e Surroca (2007). A equação 2 se refere ao modelo alternativo que não contempla a variável relativa à produção porque esse quociente tomaria valor 1 , uma vez que a produção (P) é aproximada pelo faturamento (F).

$$
\left(\frac{I I}{F}\right)_{i, t}=\beta_{1}\left(\frac{I I}{F}\right)_{i, t-1}+\beta_{2}\left(\frac{I I}{F}\right)_{i, t-1}^{2}+\beta_{3}\left(\frac{F C}{F}\right)_{i, t-1}+\beta_{4}\left(\frac{D}{F}\right)_{i, t-1}^{2}+\alpha_{i}+\delta_{t}+\varepsilon_{i, t}
$$

A dificuldade de obtenção de dados precisos sobre gastos em inovação, destacada por Ballester, Garcia-Ayuso e Livnat (2003); Hall e Oriani (2004); e Munari, Oriani e Sobrero (2005), também foi encontrada na realização deste trabalho. No entanto, como eles, tentou-se contornar essa limitação evitando que ela fosse um fator que impedisse a pesquisa. Desse modo, neste trabalho se aproximam os investimentos anuais da empresa em inovação $\left(\mathrm{II}_{t}\right)$ pela variação do ativo diferido, como feito por Crisóstomo e Vallelado (2006). Essa aproximação é possível pelo fato de que se registram no ativo diferido os valores de gastos em projetos de investimento em pesquisa, de- 
senvolvimento e inovação (P\&D\&I), de acordo com o inciso V do art. 179 da Lei no 6.404/1976 e com a seção 5.4 da Resolução CVM no 29, de 5 de fevereiro de 1986 (Brasil, 1986; Iudícibus, Martins e Gelbcke, 2003). Afora esses valores, no ativo diferido também se registram valores relativos a projetos de reorganização e gastos de implantação de projetos que também podem estar direta ou indiretamente associados à inovação, e outros elementos que contribuem para a formação do resultado de mais de um exercício social. É possível que esses outros valores possam ocasionar ruídos. No entanto, comparando-se os valores de intensidade do investimento usando esses valores com os respectivos valores publicados pelo IBGE referentes a 2003 (Brasil, 2003), verificou-se que os resultados eram qualitativamente equivalentes para o referido ano.

Assim como os gastos de capital, os gastos em inovação também estão submetidos à identidade de acumulação de capital inovação $\left(I I_{t}=K I_{t}-\right.$ $\left.(1-\zeta) K I_{t-1}\right)$, onde $K I$ é o estoque de capital em inovação aproximado pelo ativo diferido e $\zeta$ é a taxa de amortização.

Desse modo, fora a variável que mensura os gastos em inovação, todas as demais são equivalentes àquelas do modelo da equação (1), sendo no modelo da equação (2) relativizadas pelo faturamento da empresa no período $\left(F_{t}\right)$.

\section{RESULTADOS}

O primeiro estágio da pesquisa está composto por uma análise descritiva das amostras de modo a se conhecer melhor o universo investigado. A tabela 1 , já mencionada, mostra que as empresas pesquisadas estão distribuídas em 27 setores da economia. Além disso, cabe ressaltar que os modelos (equações 1 e 2) foram estimados com variáveis dummy de setores.

Para cada amostra, investimento em capital físico e em inovação, apresenta-se o número de observações anuais de empresas de cada setor, em número absoluto e relativo.

Os modelos foram estimados pelo Método Generalizado de Momentos (GMM) proposto por Arellano e Bond (1991). A metodologia de dados em painel permite o tratamento da heterogeneidade não observável constante associada aos efeitos fixos, ou seja, aquelas particularidades específicas de cada empresa e que se mantêm ao longo do tempo. Além disso, o GMM per- 
mite tratar adequadamente os problemas de endogeneidade. Por questão de análise de sensibilidade se estimou uma variação do modelo na qual se considera uma relação linear com o endividamento, como já mencionado.

A tabela 2 apresenta estatísticas básicas que descrevem as principais características da amostra. As variáveis dos modelos de investimento em capital físico (painel A) nos mostram que, em média, a intensidade de investimento em capital físico é de $11,58 \%$, estando abaixo de países desenvolvidos, como os 12,9\% de Estados Unidos (Chiao, 2002), os 13,9\% de Alemanha (Harhoff, 1998) e os 11,7\% do Reino Unido (Bond, Elston, Mairesse e Mulkay, 2003). O nível de fluxo de caixa relativamente ao estoque de capital físico apresenta uma média de $39 \%$ e a relação do faturamento com o estoque de capital físico é de aproximadamente 300\%. O endividamento bancário, medido pela relação entre dívida bancária e estoque de capital físico imobilizado, é de $151 \%$, mostrando alto endividamento médio para o conjunto de empresas.

As variáveis dos modelos de gastos em inovação (tabela 2, painel B) nos permitem ver que a intensidade de investimento em inovação, medida pela relação entre montante investido e faturamento anual, é de $0,4 \%$, o que é muito inferior ao verificado em países desenvolvidos, como era esperado. Nos EUA, se observa uma taxa equivalente de 4,0\% (Hall, Mairesse, Branstetter e Crepon, 1999); na França, de 3,62\% (Mulkay, Hall e Mairesse, 2001); e na Áustria, de 2,0\% (Gugler, 2003). Além disso, verifica-se uma razão entre fluxo de caixa e faturamento de $12,74 \%$, e um nível de endividamento bancário com relação ao faturamento de 49,56\%.

Tabela 2: Estatísticas descritivas das variáveis dos modelos

\begin{tabular}{ccccc}
\hline & \multicolumn{4}{c}{ Painel A: Variáveis relativas a investimento em capital físico } \\
\hline Variável & Média & Desvio padrão & Mínimo & Máximo \\
\hline$(I C F / K)$ & 0,11582 & 0,53842 & $-0,88235$ & 3,83156 \\
\hline$(F C / K)$ & 0,39060 & 1,13552 & $-1,27961$ & 3,13856 \\
\hline$(P / K)$ & 3,07309 & 4,05557 & 0,00000 & 15,82312 \\
\hline$(D / K)$ & 1,51578 & 3,19129 & 0,00000 & 13,79375 \\
\hline
\end{tabular}

Painel B: Variáveis relativas a investimento em inovação

\begin{tabular}{ccccc}
\hline$(I I / S)$ & 0,00414 & 0,05118 & $-0,22602$ & 0,34453 \\
\hline$(F C / S)$ & 0,12741 & 0,33671 & $-0,66795$ & 0,89671 \\
\hline$(D / S)$ & 0,49563 & 0,59991 & 0,00000 & 2,32260 \\
\hline
\end{tabular}


Os resultados das estimações para o investimento em capital físico são apresentados na tabela 3. Na ausência de dificuldades de financiamento externo, espera-se que os parâmetros estimados se comportem como previsto na hipótese de mercado perfeito, quer dizer, relação positiva do investimento em capital físico atual com o investimento anterior e a produção, indicando capacidade de manutenção da intensidade de investimento da empresa, e negativa, com os custos de ajuste de capital, que seriam os únicos moderadores do investimento. Além disso, sob a hipótese de mercado perfeito tam-

\section{Tabela 3: Estimação do modelo para investimento em capital físico}

Coeficientes estimados e desvios padrão robustos à heteroscedasticidade (entre parênteses) referentes à estimação do modelo da equação (1) para investimento em capital físico. O Painel A contém resultados para o modelo de relação quadrática do endividamento. O Painel B informa resultados do modelo de relação linear com o endividamento. Modelos estimados pelo Método Generalizado de Momentos (GMM). O teste de Sargan/Hansen de sobreidentificação de restrições segue uma distribuição $\chi^{2}$, e AR2 é o teste de autocorrelação de segunda ordem dos erros residuais. ${ }^{*}{ }^{* *} e^{* * *}$ se referem, respectivamente, a significativo em nível de $10 \%, 5 \%$ e $1 \%$.

\begin{tabular}{|c|c|}
\hline $\begin{array}{l}\text { Painel A: } \\
\text { Modelo relação quadrática com o endividamento }\end{array}$ & $\begin{array}{l}\text { Painel B: } \\
\text { Modelo relação linear com o endividamento }\end{array}$ \\
\hline$\left(\frac{I C F}{K}\right)_{i, t}=\beta_{1}\left(\frac{I C F}{K}\right)_{i, t-1}+\beta_{2}\left(\frac{I C F}{K}\right)_{i, t-1}^{2}+\beta_{3}\left(\frac{F C}{K}\right)_{i, t-1}+\beta_{4}\left(\frac{P}{K}\right)_{i t-1}+\beta_{5}\left(\frac{D}{K}\right)_{i, t-1}^{2}+a_{i}+\delta_{t}+\varepsilon_{i, t}$ & $\left(\frac{I C F}{K}\right)_{i, t}=\beta_{1}\left(\frac{I C F}{K}\right)_{i, t-1}+\beta_{2}\left(\frac{I C F}{K}\right)_{i, t-1}^{2}+\beta_{3}\left(\frac{F C}{K}\right)_{i, t-1}+\beta_{4}\left(\frac{P}{K}\right)_{i, t-1}+\beta_{5}\left(\frac{D}{K}\right)_{i, t-1}+\alpha_{i}+\delta_{t}+\varepsilon_{i, t}$ \\
\hline Coef./(d.p.) Signif. & Coef./(d.p.) Signif. \\
\hline$(\mathrm{ICF} / \mathrm{K})_{t-1}$ & $(\mathrm{ICF} / \mathrm{K})_{t-1}$ \\
\hline$(0,080)$ & $(0,079)$ \\
\hline$-0,0672$ ** & $-0,0733 * *$ \\
\hline$(0,029)$ & $(0,029)$ \\
\hline $0,0421 * *$ & $0,0493 * *$ \\
\hline$(0,022)$ & $(0,023)$ \\
\hline $0,0276 * * *$ & $0,0270 * * *$ \\
\hline$(0,009)$ & $(0,009)$ \\
\hline$(D / S)^{2} t-1$ & $(D / S)_{t-1}$ \\
\hline$(0,001)$ & $(0,012)$ \\
\hline Constante & Constante \\
\hline$(0,697)$ & $(0,708)$ \\
\hline N. obs. & 2.808 \\
\hline N. empresas & 289 \\
\hline 1,98 & 2,31 \\
\hline (valor $p)$ & 0 \\
\hline AR2 (valor $p$ ) & 0,948 \\
\hline Sargan/Hansen & $285,12(261)$ \\
\hline (valor $p)$ & 0,146 \\
\hline
\end{tabular}


bém se espera ausência de correlação, ou correlação negativa, da intensidade de gastos em investimento com o fluxo de caixa, pois a empresa não dependerá de fundos internos para executar seus projetos. Por fim, na ausência de dificuldades de financiamento externo, espera-se uma correlação positiva do investimento com o endividamento, pois a empresa não terá dificuldades para obter financiamento externo para viabilizar seus bons projetos de investimento. Entretanto, na presença de restrições financeiras decorrentes de imperfeições de mercado, outra situação pode apresentar-se. Nesse caso, se verificaria a presença de dificuldades de financiamento externo que se evidenciariam através de uma correlação positiva entre investimento e fundos gerados internamente, como amplamente considerado na literatura. Além disso, também se podem verificar outros sinais indicativos de dificuldades de financiamento externo, como a ausência de capacidade de persistência na política de investimento evidenciado por uma correlação negativa entre investimento atual e prévio, e também a ausência de correlação, ou mesmo correlação negativa, entre o investimento e o endividamento.

De fato, observando-se os resultados da tabela 3, pode-se dizer que a dinâmica teórica do modelo de custos de ajuste a favor da hipótese nula de mercado perfeito de ausência de dificuldade de financiamento externo é rejeitada, pois se verificou um efeito positivo e significativo do fluxo de caixa sobre o investimento em capital físico nos dois modelos estimados (relação quadrática e linear com o endividamento bancário), o que é um sinal de que as empresas têm dificuldade de acesso ao crédito bancário. Esses resultados confirmam e dão robustez aos de Terra (2003) que estão na mesma direção, bem como a evidência internacional comentada na seção 2 .

Na tabela 4 se podem ver os resultados dos modelos estimados para os investimentos em inovação. Para estes também se pode recusar a hipótese estatística de ausência de dificuldade de financiamento externo pela latente influência do fluxo de caixa sobre os investimentos em inovação. Esses resultados também estão alinhados com a evidência internacional vista na seção 2.

Além da correlação positiva dos dois tipos de investimentos com o fluxo de caixa, um outro fator dá mais força à hipótese alternativa de presença de restrições financeiras. Como já comentado, teoricamente se espera uma re- 


\section{Tabela 4: Estimação do modelo para investimento em inovação}

Coeficientes estimados e desvios padrão robustos à heteroscedasticidade (entre parênteses) referentes à estimação do modelo da equação (2) para investimento em inovação. O Painel A contém resultados para o modelo de relação quadrática do endividamento. O Painel B informa resultados do modelo de relação linear com o endividamento. Modelos estimados pelo Método Generalizado de Momentos (GMM). O teste de Sargan/Hansen de sobreidentificação de restrições segue una distribuição $\chi 2$, e AR2 é o teste de autocorrelação de segunda ordem dos erros residuais. ${ }^{*},{ }^{*} \mathrm{e}^{* * *}$ se referem, respectivamente, a significativo em nível de $10 \%, 5 \%$ e $1 \%$.

\begin{tabular}{|c|c|}
\hline $\begin{array}{l}\text { Painel A: } \\
\text { Modelo relação quadrática com o endividamento }\end{array}$ & $\begin{array}{l}\text { Painel B: } \\
\text { Modelo relação linear com o endividamento }\end{array}$ \\
\hline$\left(\frac{I I}{F}\right)_{i, t}=\beta_{1}\left(\frac{I I}{F}\right)_{i, t-1}+\beta_{2}\left(\frac{I I}{F}\right)_{i, t-1}^{2}+\beta_{3}\left(\frac{F C}{F}\right)_{i, t-1}+\beta_{4}\left(\frac{D}{F}\right)_{i, t-1}^{2}+\alpha_{i}+\delta_{t}+\varepsilon_{i, t}$ & $\left(\frac{I I}{F}\right)_{i, t}=\beta_{1}\left(\frac{I I}{F}\right)_{i, t-1}+\beta_{2}\left(\frac{I I}{F}\right)_{i, t-1}^{2}+\beta_{3}\left(\frac{F C}{F}\right)_{i, t-1}+\beta_{4}\left(\frac{D}{F}\right)_{i, t-1}+\alpha_{i}+\delta_{t}+\varepsilon_{i, t}$ \\
\hline Coef./(d.p.) Signif. & Coef./(d.p.) Signif. \\
\hline$-0,1661 * * *$ & $-0,1596 * * *$ \\
\hline$(0,044)$ & $(0,044)$ \\
\hline$(\mathrm{II/S})^{2} t-1$ & 0,6170 * \\
\hline$(0,393)$ & $(0,355)$ \\
\hline $0,0220 * *$ & 0,0258 ** \\
\hline$(0,011)$ & $(0,011)$ \\
\hline$(\mathrm{D} / \mathrm{S})^{2}{ }_{t-1}$ & $-0,0020$ \\
\hline$(0,003)$ & $(0,007)$ \\
\hline Constante & Constante \\
\hline$(0,035)$ & $(0,038)$ \\
\hline N. obs. & 2023 \\
\hline N. empresas & 206 \\
\hline 2,04 & 1,96 \\
\hline (valor $p)$ & 0,011 \\
\hline $\mathrm{AR}^{2}$ (valor $p$ ) & 0,809 \\
\hline$\underline{\text { Sargan/Hansen }}$ & $166,03(164)$ \\
\hline (valor $p)$ & 0,441 \\
\hline
\end{tabular}

lação positiva entre o investimento atual e o anterior no sentido de que indica capacidade de persistência de investimento da empresa. A ausência dessa correlação é sinal de falta de capacidade da empresa em seguir investindo, o que pode ser resultado das dificuldades de financiamento. No caso do investimento em capital físico (tabela 3), essa correlação não é significativa como esperado pela hipótese de mercado perfeito. Ao observar-se as estimações para investimento em inovação (tabela 4), essa correlação é negativa e muito significativa, demonstrando que esse coletivo de empresas tem dificuldade em manter seu nível de investimento em inovação, o que 
pode ser consequência das dificuldades de financiamento externo, como previsto teoricamente ser mais provável para esse tipo de investimento (Hall, 2005). Essa dificuldade de financiamento externo para investir em inovação está na mesma direção da evidência internacional, apesar de, no Brasil, Jensen, Menezes e Sbragia (2004) não terem encontrado poder explicativo para o lucro líquido sobre os gastos em inovação.

Finalmente, sobre os aspectos econométricos, cabe ressaltar que as duas condições necessárias para a validade das estimações feitas pelo método GMM foram satisfeitas para todos os modelos. Primeiramente, o teste de Arellano-Bond (AR2) não permitiu o descarte da hipótese nula de ausência de autocorrelação de segunda ordem dos erros residuais. Segundo, o teste de Sargan/Hansen de existência de sobreidentificação de restrições não permitiu a rejeição da hipótese estatística de que cada modelo está bem identificado. Dessa forma, os resultados desses testes permitem considerar que todas as estimações são válidas e, assim, satisfazem os requisitos estatísticos requeridos para habilitar os resultados das estimações às análises realizadas.

Em função desses resultados, podem-se confirmar as duas hipóteses de pesquisa propostas e responder à pergunta de investigação feita na introdução. De fato, encontrou-se respaldo para a hipótese 1, de que a empresa brasileira encara dificuldades de financiamento externo para seu investimento em capital físico, o que ficou patente pela alta sensibilidade desses gastos à disponibilidade de fundos internos. O mesmo se pode dizer com relação à hipótese 2 proposta, de que a empresa brasileira encara dificuldades de financiamento externo para financiar seu investimento em inovação, que foi confirmada também pela revelação de uma correlação positiva entre esse investimento e o fluxo de caixa. Desse modo, pode-se responder positivamente à pergunta de pesquisa proposta: a empresa brasileira tem dificuldades para financiar com fundos externos tanto seu investimento em capital físico como em inovação.

\section{CONCLUSÕES}

Este trabalho apresenta resultados que se somam à evidência internacional sobre dificuldades de financiamento externo, para investimento em capital físico e em inovação, de empresas em distintos mercados, inclusive Brasil 
(Moreira e Puga, 2000; Terra, 2003). Os resultados permitem considerar que a empresa brasileira, de fato, encara dificuldade de financiamento externo para seu investimento em capital físico e em inovação, como se verificou pela alta influência dos fundos internos da empresa na intensidade de investimento em capital físico e em inovação. Os problemas de assimetria informativa entre empresa e mercado de financiamento parecem ser relevantes no Brasil juntamente com a pouca proteção aos credores externos, o que deve contribuir para essa dependência de fundos internos para esses investimentos essenciais ao desenvolvimento da empresa. Esses novos resultados foram encontrados através da aplicação de um modelo de investimento dinâmico baseado na equação Euler, aplicado com sucesso em diferentes mercados, que leva em conta os custos de ajuste de capital, o nível de produção, a situação de liquidez da empresa e o endividamento bancário, como possíveis fatores explicativos da intensidade de investimento em capital físico e em inovação. Destaca-se também que os resultados são robustos à variação nos modelos, considerando relação quadrática e linear com o endividamento bancário.

Algumas contribuições deste trabalho podem ser destacadas. Trata-se de uma pesquisa sobre dificuldades de financiamento externo em um mercado em desenvolvimento, o que é escasso na literatura, que está mais concentrada em países desenvolvidos. Também se deve mencionar a aplicação de um modelo de investimento robusto aplicado para a empresa brasileira especificamente. Em especial, destaca-se a contribuição de pesquisa sobre as dificuldades de financiamento para o investimento em inovação, que é ainda mais escassa em mercados em desenvolvimento em geral, e no Brasil especificamente. Do ponto de vista acadêmico, este trabalho apresenta a contribuição do uso de técnicas de estimação modernas e mais adequadas para o problema investigado, que é a metodologia de dados em painel e Método Generalizado de Momentos (GMM), uma necessidade apontada por Moreira e Puga (2000). A partir de um ponto de vista político, pode-se considerar que esses resultados indicam a necessidade de continuidade de mudanças estruturais no mercado brasileiro que permitam o fortalecimento do mercado de capitais como possível caminho para reduzir o problema de restrições financeiras das empresas que operam nesse ambiente. 


\section{REFERÊNCIAS BIBLIOGRÁFICAS}

AKERLOF, G. A. The market for lemons: quality uncertainty and the market mechanism. The Quarterly Journal of Economics, v. 84, n. 3, p. 488-500, 1970.

ALDRIGHI, D. M.; MAZZER NETO, R. Ownership and control in brazilian limited liability companies. Revista de Economia Política, v. 25, n. 2, 2005.

ALTI, A. How Sensitive is investment to cash flow when financing is frictionless? The Journal of Finance, v. 58, n. 2, p. 707-722, 2003.

ARELLANO, M.; BOND, S. Some tests of specification for panel data: Monte Carlo evidence and an application to employment equations. Review of Economic Studies, 58, p. 277-297, 1991.

AUDRETSCH, D. B.; WEIGEND, J. Do knowledge conditions make a difference?: Investment, finance and ownership in German industries. Research Policy, v. 34, Issue 5, june 2005, p. 595-613, 2005.

BALLESTER, M.; GARCIA-AYUSO, M.; LIVNAT, J. The economic value of the R\&D intangible asset. European Accounting Review, v. 12, n. 4, p. 605-633, 2003.

BECHT, M., RÖELL, A. Blockholdings in Europe: an international comparison. European Economic Review, v. 43, Issues 4-6, apr. 1999, p. 1049-1056, 1999.

BECK, T.; DEMIRGUÇ-KUNT, A.; LEVINE, R. Law, endowments, and finance. Journal of Financial Economics, v. 70, Issue 2, nov. 2003, p. 137-181, 2003.

BOND, S.; MEGHIR, C. Dynamic investment models and the firm's financial policy. The Review of Economic Studies, v. 61, Issue 2, 197-222, 1994.

— ELSTON, J. A.; MAIRESSE, J.; MULKAY, B. Financial factors and investment in Belgium, France, Germany, and the United Kingdom: a comparison using company panel data. The Review of Economics and Statistics, v. 85, n. 1, feb. 2003.

BOND, S.; VAN REENEN, J. HARHOFF, D.; Investment, R\&D and Financial Constraints in Britain and Germany. The Institute of Fiscal Studies, IFS Working Paper W99/05, 55p. UK, oct. 2003.

Microeconomic Models of Investment and Employment. The Institute of Fiscal Studies, 130p., UK, dec. 2003. (mimeos.)

BOUGHEAS, S.; GÖRG, H.; STROBL, E. Is R\&D financially constrained? Theory and evidence from irish manufacturing. Review of Industrial Organization. Boston: v. 22, Iss. 2; p. 159, mar. 2003.

BRASIL. DELIBERAÇÃO CVM n. 29, de 5 fev. 1986.

. Pesquisa Industrial de Inovação Tecnológica. INSTITUTO BRASILEIRO DE GEOGRAFIA E ESTATÍSTICA - IBGE, 2003.

BROWN, W. R\&D intensity and finance: are innovative firms financially constrained? Discussion Paper dp 271, London School of Economics, Financial Markets Group, 1997.

CABALLERO, RICARDO J. Aggregate investment. In: TAYLOR, JOHN B. e WOODFORD, MICHAEL (eds.), Handbook of Macroeconomics, v. 1B, Elsevier, North-Holland, Amsterdam, 1999. 
CARPENTER, R. E.; FAZZARI, S. M.; PETERSEN, B. C. Inventory investment, internal-finance fluctuations, and the business cycle. Brookings Papers on Economic Activity. Washington: 1994. n. 2, p. 75-122, 1994.

CHIAO, Chaoshin relationship between debt, R\&D and physical investment, evidence from US firm-level data. Applied Financial Economics, v. 12, p. 105-121, 2002.

CHIRINKO, R. S. Business fixed investment spending: modelling strategies, empirical and policy implications. Journal of Economic Literature, 31, p. 1875-1911, 1993a.

-; SCHALLER, H. Why Dões Liquidity Matter in Investment Equations? Journal of Money, Credit \& Banking v. 27, n. 2, may, 1995, p. 526 (23), 1995.

CRISÓSTOMO, V. L.; VALLELADO, E. Possível reação do mercado brasileiro aos investimentos em P\&D. Revista de Administração Mackenzie, v. 7, n. 1, p. 96-114, São Paulo, 2006 (http:// www.mackenzie.br/editoramackenzie/revistas/administração/), 2006b.

CROTTY, J. R. Neoclassical and Keynesian approaches to the theory of investment. Journal of Post Keynesian Economics, 14, 4, 483-496, 1992.

CUERVO, A. Corporate governance mechanisms: a plea for less code of good governance and more market control. Corporate Governance: An International Review, v. 10, Issue 2, p. 84-93, april 2002.

. El Gobierno de la empresa: un problema de conflicto de intereses. In: BUENO C. (dir. e coord.). El gobierno de la empresa. En busca de la transparencia e la confianza, p. 115-135, 2004.

DEGRYSE, H. A.; DE JONG, A. Investment and internal finance: asymmetric information or managerial discretion?. International Journal of Industrial Organization, v. 24, Issue 1, jan. 2006, p. 125-147, 2006.

DE LONG, J. B.; SUMMERS, L. H. Equipment investment and economic growth. NBER Working Paper n. W3515, nov. 1983, 1990.

FAZZARI, S. M.; HUBBARD, R. G.; PETERSEN, B. C. Financing Constraints and Corporate Investment. Brookings Papers on Economic Activity. p. 141 (66 p.), Washington: 1988.

GALEOTTI, M.; SCHIANTARELLI, F.; JARAMILLO, F. Investment decisions and the role of debt, liquid assets and cash flow: evidence from Italian panel data. Applied Financial Economics. v. 4, n. 2, p. 121-132, 1994.

GÉRARD, M.; VERSCHUEREN, F. Finance, uncertainty and investment: assessing the gains and losses of a generalized non linear structural approach using Belgian panel data. National Bank of Belgium Working Paper - Research Series (WP26), 2002.

GOERGEN, M.; RENNEBOOG, L. Investment policy, internal financing and ownership concentration in the UK. Journal of Corporate Finance, v. 7, Issue 3, p. 257-284, sep. 2001.

GREENWALD, B.; STIGLITZ, J. E.; WEISS, A. Informational imperfections in the capital market and macroeconomic fluctuations. The American Economic Review. Nashville: may 1984. v. 74, Iss. 2; p. 194 (6 p.), 1984.

GUGLER, K. Corporate governance, dividend payout policy, and the interrelation between dividends, R\&D, and capital investment. Journal of Banking \& Finance, v. 27, Issue 7, July 2003, p. 1.297-1.321, 2003. 
-; MUELLER, D. C.; YURTOGLU, B. B. Marginal q, Average q, Cash Flow and Investment. Southern Economic Journal, v. 70, n. 3, p. 512-531, 2004.

HALL, B. H. Investment and research and development at the firm level: does the source of financing matter? NBER Working Paper Series 4096, june, 1992.

The Financing of Innovation. Shane, S., ed., Blackwell Handbook of Technology and Innovation Management, Oxford: Blackwell Publishers, Ltd., 2005.

; ORIANI, R. Does the market value R\&D investment by european firms? Evidence from a panel of european firms in France, Germany and Italy. NBER Working Paper Series 10048. March 2004.

- ; MAIRESSE, J.; BRANSTETTER, L.; CREPON, B. Does cash flow cause investment and R\&D: an exploration using panel data for French, Japanese and United States firms in the scientific sector. In: D. AUDRETSCH AND A. R. THURIK, eds., Innovation, Industry Evolution and Employment (Cambride University Press, Cambridge), 1999.

HARHOFF, D. Are there financing constraints for innovation and investment in German manufacturing firms? Annales d'Économie et de Statistique, 49/50, 421-456, 1998.

HENNESSY, C. A.; LEVY, A.; WHITED, T. M. Testing Q theory with financing frictions. Journal of Financial Economics, v. 83, Issue 3, mar. 2007, p. 691-717, 2007.

HUBBARD, R. G. Capital-market imperfections and investment. Journal of Economic Literature. v. 36, Iss. 1; p. 193 (33 p.), Nashville: mar. 1998.

; KASHYAP, A. K.; WHITED, T. M. Internal finance and firm investment. Journal of Money, Credit, and Banking. Columbus: Aug. 1995. v. 27, Iss. 3; p. 683 (19 p.), 1995.

IUDÍCIBUS, S. de; MARTINS, E.; GELBKE, E. R. Manual de contabilidade das sociedades por ações". Atlas, 6. ed. São Paulo, 2003, 2003.

JENSEN, M. C.; MECKLING, W. H. Theory of the firm: managerial behavior, agency cost and ownership structure. Journal of Financial Economics, oct. 1976, v. 3, n. 4, p. 305-360, 1976.

JENSEN, J.; MENEZES, N. F. E SBRAGIA, R. Os determinantes dos gastos em P\&D no Brasil: uma análise com dados em painel. Estudos Econômicos, São Paulo, v. 34, n. 4, 661-691, out.dez. 2004.

KAPLAN, S. N.; ZINGALES, L. Do investment-cash flow sensitivities provide useful measures of financing constraints? Quarterly Journal of Economics, v. 112, n. 1 (feb. 1997): p. 169 (47), 1997.

LA PORTA, R.; LÓPEZ-DE-SILANES, F.; SHLEIFER, A.; VISHNY, R. W. Legal Determinants of External Finance. Journal of Finance, v. 52, n. 3, p. 1131-1150, 1997.

Law and Finance. Journal of Political Economy, v. 106, n. 6, p. 1.113-1.155, dec. 1998.

Investor protection and corporate governance. Journal of Financial Economics, v. 58, Issues 1-2, 2000, p. 3-27, 2000. 
LÍZAL, L.; SVEJNAR, J. Investment, credit rationing, and the soft budget constraint: evidence from Czech Panel Data. The Review of Economics and Statistics, v. 84, n. 2, p. 353-370 (may. 1, 2002), 2002.

LUCAS, R. E. Adjustment costs and the theory of supply. The Journal of Political Economy, v. 75, n. 4, Part 1 (aug. 1967), p. 321-334, 1967.

MARQUES, A. M.; FOCHEZATTO, A. Taxa de juros e prêmio de risco: investigando a hipótese Bresser-Nakano para a economia brasileira, 1995-2005. Revista de Economia Contemporânea, v. 11, n. 2. Rio de Janeiro, may.-aug. 2007.

MODIGLIANI, F.; MILLER, M. H. The cost of capital, corporation finance and theory of investment. The American Economic Review, v. 48, n. 3, jun., 1958, p. 261-297, 1958.

MORCK, R.; WOLFENZON, D.; YEUNG, B. Corporate Governance, Economic Entrenchment, and Growth. Journal of Economic Literature. Nashville: sep. 2005. v. 43, Iss. 3; p. 655 (66 p.), 2005.

MOREIRA, M. M.; PUGA, F. P. Como a indústria brasileira financia o seu crescimento: uma análise do Brasil pós-Real. Revista de Economia Contemporânea, Rio de Janeiro, v. 5 (ed. especial), p. 1-39, out. 2000.

MOYEN, N. Investment-Cash Flow Sensitivities: constrained versus unconstrained Firms. The Journal of Finance, 59 (5), 2061-2092, 2004.

MULKAY, B.; HALL, B. H.; MAIRESSE, J. Firm level investment and R\&D in France and the United States. In: HERRMANN, HEINZ; ROLF S. (eds.), Investing Today for the World of Tomorrow, Springer Verlag, 2001.

MUNARI, F.; ORIANI, R.; SOBRERO, M. The effects of owner idendity and financial markets on R\&D investments: a study of western european firms. Available at SSRN: http://ssrn. com/abstract=717684, nov. 2005.

MYERS, S. C. Financing of Corporations. In: CONSTANTINIDES, G.; HARRIS, M.; STULZ, R. M. eds., Handbook of the Economics of Finance. Elsevier, North-Holland, Amsterdam, p. 215253, 2003.

MAJLUF, N. S. Corporate Financing and investment decisions when firms have information that investors do not have. Journal of Financial Economics, v. 13, n. 2, 1984, p. 187-221, 1984.

SHLEIFER, A.; VISHNY, R. W. A Survey of corporate governance. Journal of Finance, v. 52 Issue 2, p. 737-783, june, 1997.

STEIN, JEREMY C. Agency, information, and corporate investment. In: CONSTANTINIDES, G.; HARRIS, M.; STULZ, R. M. eds., Handbook of the Economics of Finance. Elsevier, NorthHolland, Amsterdam, p. 101-141, 2003.

STIGLITZ, J. E.; WEISS, A. Credit rationing in markets with imperfect information. The American Economic Review. Nashville: jun. 1981, v. 71, Iss. 3; p. 393 (17 p.), 1981.

STUDART, R. Financial opening and deregulation in Brazil in the 1990s moving towards a new pattern of development financing? The Quarterly Review of Economics and Finance, v. 40, Issue 1, Spring 2000, p. 25-44, 2000. 
TERRA, M. C. T. Credit Constraints in Brazilian firms: evidence from panel data. Revista Brasileira de Economia, v. 57, n. 2, p. 443-464, 2003.

TRIBO, J. A.; BERRONE, P.; SURROCA, J. Do the type and number of blockholders influence R\&D investments? New evidence from Spain. Corporate Governance: An International Review 15 (5), 828-842, 2007.

VOGT, S. C. The cash flow/investment relationship: evidence from US manufacturing firms. Financial Management. v. 23, Iss. 2, p. 3; Tampa: Summer 1994.

WHITED, T. M. Debt, Liquidity constraints, and corporate investment: evidence from panel data. Journal of Finance, p. 1.425-1.460, sep. 1992. 